\title{
Herpes simplex virus infections, Pathophysiology and Management
}

\author{
Murtaza Mustafa ${ }^{1}$, EM.Illzam ${ }^{2}$, RK.Muniandy ${ }^{3}$, AM.Sharifah ${ }^{4}$, \\ MK.Nang ${ }^{5}$, B.Ramesh ${ }^{6}$ \\ ${ }^{1,3,5}$, Faculty of Medicine and Health Sciences, University Malaysia Sabah, Kota Kinabalu, \\ Sabah, Malaysia. \\ 2. Clinic Family Planning Association, Kota Kinabalu, Sabah, Malaysia. \\ ${ }^{4}$ Quality Unit, Hospital Queen Elizabeth, Kota Kinabalu, Sabah, Malaysia. \\ ${ }^{6}$ Sarawak Ministry of Health, Clinic, Bintulu, Sarawak, Malaysia.
}

\begin{abstract}
Herpes is caused by Herpes simplex virus (HSV).HSV-1causes orofacial and genital infections.HSV-2 causes primarily genital infections. In the U.S.HSV-1 is more prevalent than HSV-2, and HSV-2 is predominant among American African, and women. Initial HSV infection is often subclinical. Viral replication occurs in ganglia, virus spreads to other mucosal surfaces through peripheral sensory nerves. Clinical manifestation include, skin and mucosal infection, orofacial herpes, genital herpes, herpetic whitlow, herpes encephalitis, neonatal herpes acquired at the time of delivery, although rare but serious condition and herpes keratitis damage the eyes. Diagnostic tests include, culture, antibodies detection, skin biopsy, and PCR for presence of viral DNA.Topical anesthetic treatment to relieve itching and pain. Antiviral drugs such as acyclovir,valaciclivir and famiciclovir are effective. Antiviral for herpes suppressive therapy is recommended during the last months of pregnancy. Recurrent outbreaks are common and vary between individuals; genetics play a role in the frequency of cold sore outbreaks. Development of a drug to block the microRNA whose job is it to suppress HSV-1 into latency, may prevent the outbreaks. Prevention with antiviral medication in conjunction with a condom.
\end{abstract}

Key words: Herpes, Herpes simplex virus (HSV),HSV-1,HSV-2,Management, and Prognosis

\section{Introduction}

Herpes simplex viruses have worldwide distribution and are found in the most remote human populations [1].Worldwide rates of either HSV-1 or HSV-2 are between 60 and 95\% in adults.HSV-1 is more common than HSV-2, with rates increasing as people age[2].HSV-1 rates are between $70 \%$ and $80 \%$ in populations of low socioeconomic status and $40 \%$ to $60 \%$ in populations of improved socioeconomic status[2].Prevalence of HSV-2 in those between the ages of 15 and 50 is about 535 million as of 2003 or $16 \%$ of the population, with highest rates in in sub-Sahara Africa and lowest in western Europe, and with greater rates among women and those in the developing world[3,4].In the U.S,57.7\% of the population is infected with HVS1 and $16.2 \%$ are infected with HVS-2,and prevalence of HVS-2 was $39.2 \%$ in blacks and $20.9 \%$ in women[5,6].Herpes infection is the predominant cause of genital ulcers throughout the world, with increased use of HSV PCR for detection of HSV infection,[1].HVS-1 causes primarily mouth, throat, face, eye, and central nervous system infections, whereas HSV-2 causes primarily anogenital infections. However, each may cause infections in all areas [2].HSV infection is contracted through direct contact with an active lesion or body fluid of an infected person[7].Clinical presentations of HSV include, skin and mucosa infection, orofacial herpes, genitalherpes,herpeticwhitlow, herpes encephalitis, cognitive deficits of bipolar disorder and Alzheimer's disease[8].Diagnosis by detection of HSV antibodies by PCR[1], and the new Immunodot glycoprotein G-specific(IgG) test is more than $98 \%$ specific at discriminating HSV-1 from HSV2[9].Management by antiviral medications, can reduce the frequency, and topical anesthetic to relieve itching and pain[10,].Prevention by barriers such as condom, and the use of antiviral agents. The paper reviews the current literature, pathophysiology, and management of Herpes simplex infections.

\section{Historical perspective}

The word herpes (from Greek, "to creep") has been used in medicine since antiquity. Cold sores (herpes febirilis) were described by the Romans physician Herodotus in AD100[11]Emperor Tiberius is said to have banned kissing in Rome for a time to so many people having cold sores. In the $16^{\text {th }}$ century Romeo and Juliet, blisters"o'er ladies" lips are mentioned[12].Genital herpes was first described by John Astruc, physician to king of France in 1736:the first English translation appeared in his treatise on venereal disease in1754[13].In $18^{\text {th }}$ century, it was so common among prostitutes, it was called "a vocational disease of women"[12].The term 
“herpes appeared in Richard Boultn'sA system of Rational and Practical Chirurgery in 1713, where the terms "herpesmiliaris" and 'herpes exedens' also appeared. Herpes was not found to be a virus until the 1940s [12].Infection in orolabial lesions was transmitted to other humans in the late $19^{\text {th }}$ century. The disease was successfully transferred to rabbits in the early $20^{\text {th }}$ century, and HSV was grown in vitro in 1925[14].In the 1960's Nahmias and Dowdle reported two antigenic types of HSV with different sites of viral infection [15].

Herpes antiviral therapy began in the early 1960s with the experimental use of medications that interfered with viral replication called deoxyribonucleic acid (DNA) inhibitors. The original use was against normally fatal or debilitating illnesses such as encephalitis, keratitis in immunocompromised transplant patients) or disseminated herpes zoster[16-19].The introduction of 9- $\beta$-D arabinofuranosyladenine (ara-A or vidarabine),considerably less toxic than ara-C, in mid-1970s, heralded the way for the beginning of regular neonatal antiviral treatment. Vidarabine was the first systematically administered antiviral medication with activity against HSV for which therapeutic efficacy outweighed toxicity for the management of life-threatening HSV disease[20].The introduction of9-(2-hydroxyethoxmethyl)guanine,AKA acyclovir in the late 1970, raised antiviral treatment another notch and led to vidarabine vs acyclovir trials in the late 1980s[21,22].The lower toxicity and ease of administration over vidarabine has led to acyclovir becoming the drug of choice for herpes treatment after it was licensed by FDA[23].Another advantage in the treatment of neonatal herpes included greater reductions in mortality and morbidity with increased dosages, which did not occur when compared with increased dosages ofvidarabine[23]. However, acyclovir seems to inhibit antibody response, and newborns on acyclovir antiviral treatment experienced a slower rise in antibody titer than those on vidarabine[23].

Herpes simplex was not always stigmatized. It was merely a cold sore in an unusual place until 1970s[24].Since the creation of herpes hype,some people experience negative feelings related to the condition following diagnosis, in particular if they have acquired the genital form of disease. Feelings can include depression, fear of rejection, feelings of isolation, fear of being found out, and self-destructive feelings [25].People with herpes virus are often hesitant to divulge to other people, including friends and family, that they are infected. This is especially true of new or potential sexual partners whom they consider casual [26].

\section{Transmission}

In 1921,Lipschutz inoculated materials from genital herpetic lesions into skin of humans, eliciting clinical infection within 48 to 72 hours in six persons and within 24 hours in one case[27]Transmission of HSV infections most frequently occurs through close contact with a person who is shedding virus at a peripheral site, at a mucosal surface, or in genital or oral secretions[28].Infection occurs by inoculation of virus onto susceptible mucosal surfaces(e.g. Oropharynx, cervix, conjunctivae) or through small cracks in the skin. Because HSV is readily inactivated at room temperature and by drying, aerosol and fomite spread are unusual means of transmission [29]. Transmission of HSV-1 from orogenital contact is increasingly recognized, perhaps because of a decrease in the age-specific prevalence of HSV-1 at the time of sexual activity starts[30].Spread of HSV-1 infection from oral secretions to other skin areas is a hazard of certain occupations(e.g., dentists, respiratory care unit personnel), and laboratory acquired and nosocomial outbreaks in hospital or nursery personnel have been reported[29]. Outbreaks among wrestlers are recognized, transmission of HSV can occur in infants born to mother excreting HSV at delivery[31,32].Anal and perianal infections with HSV-1 or HSV-2 are common among sexually active MSM (men sex with men) populations. The majority of cases occurs within 5 days of contact, highlighting the short incubation period of primary infection [33].

For HIV infection, a clear relationship between genital and plasma HIV viral load and per coital risk of HIV transmission is established [34]. However, because genital HSV-2 levels fluctuate rapidly over time, the degree to which source partner viral load during sex affects the likelihood of transmission is unknown[35].Subclinical or asymptomatic shedding of HIV in oral and genital secretions is common even in immunocompetent persons, and transmission occurs more commonly during asymptomatic shedding[28]. Frequency of detectable shedding markedly heterogeneous among those seropositive with HSV - 2,suggesting that per coital transmission may be highly dependent on the source partner [36].A modeling study predicted that a core group of "super spreaders" with high reactivation rates may account for a disproportionately large percentage of new infections[37].

\section{Pathophysiology}

Exposure to HSV at mucosal surfaces or abraded skin sites permits entry of the virus and initiation of its replication in cells of the epidermis and dermis [38].Initial HSV infection is often subclinical, without apparent lesions. In animal models and human subjects, both clinical acquisition and subclinical acquisition are associated with sufficient viral replication to permit infection of either sensory or autonomic nerve endings[38].After trans versing the neuroepithelial gap and entering the neuronal cell, the virus or, more likely the nucleocapsid is transported intra-axonally to the nerve cell bodies in ganglia[39].For HSV-1 infection, trigeminal ganglia are most commonly infected, although extension to the inferior and superior cervical ganglia 
also occurs[40].With genital infection sacral nerve root ganglia(S2 to S5) are most commonly affected. Inhumans, the interval from inoculation of virus to peripheral tissue to spread to the ganglia is unknown [41].

Viral replication occurs in ganglia and contagious neural tissue during primary infection only [38]After initial inoculation of the neural ganglion, virus spreads to other mucosal skin surfaces by centrifugal migration of infectious virions through peripheral sensory nerves. This mode of spread explains the characteristics development of new lesions distant from the initial crop of vesicles in patients with primary genital or orofacial HSV infection, the large surface area over which these vesicles may be visualized, and the recovery of virus from neural tissue distant from neurons innervating the inoculation [42].Contagious spread of virus may also take place via autoinoculation and allow further extension of disease. Viremia is present during approximately $25 \%$ of primary HSV-2 infections, and its presence may affect the natural history of HSV-2 disease in terms of site, severity and frequency of reactivation(43].Recent studies suggest that rate of reactivation is far more frequent and dynamic than previously recognized[36].The use of daily anogenital PCR swabs showed that the median shedding rate of the $95 \%$ of patients with a positive HSV-2 antibody who shed virus is $25 \%$ of days, with a wide range of interpretation variability(range, $2 \%$ to $75 \%$ )[44].Clinical studies demonstrate that host factors also influence a reactivation. Immunocompromised patients have more severe disease [45].

Antibodies that develop following an initial infection with type of HSV prevents reinfection with type same virus type-a person with a history of orofacial infection caused by HSV-1 cannot contract herpes whitlow or a genital infection caused by HSV-1.In a monogamous couple, a seronegative female runs a greater than $30 \%$ per year risk of contracting an HSV infection from a seropositive male partner[46].If an oral HSV-1 infection is contracted first, seroconversion will have occurred after 6 weeks to provide protective antibodies against a future HSV-1 infection. Herpes simplex is a double-stranded DNA virus [47].

\section{Clinical Manifestation}

HSV infection causes several distinct medical disorders. Common infection of the skin or mucosa may affect the face and mouth (orofacial herpes), genitalia (genital herpes),or hands (herpetic whitlow).More serious disorders occur when the virus infects and damage the eyes(herpes keratitis),or invades the central nervous system, damaging the brain(herpes encephalitis).People with immature or suppressed immune systems, such as newborns, transplant recipients, or people with AIDS, are prone to severe complications from HSV infections. HSV infection has also been associated with cognitive deficit of bipolar disorder, and Alzheimer's disease, although this often dependent on the genetics of the infected person [14].

In all cases, HSV never removed from the body by the immune system. Following a primary infection, the virus enters at the site of primary infection, migrates to cell body of the neuron, and becomes latent in the ganglion [27].As a result of primary infection; the body produces antibodies to particular type of HSV involved, preventing a subsequent infection of that type at a different site. In HSV-1-infected individuals, seroconversion after an oral infection prevents additional HSV-1 infections such as whitlow, genital herpes, and herpes of the eye. Prior HSV-1 seroconversion seems to reduce the symptoms of later HSV-2 infection, although HSV-2 can still be contracted. Many people infected with HSV-2 display no physical symptoms-individuals with no symptoms are described as asymptomatic or as subclinical herpes [48].

Neonatal herpes simplex is a HSV infection in an infant. It is a rare but serious condition, usually caused by vertical transmission of ( HSV-1or 2) from mother to newborn. During immunodeficiency ,herpes simplex can cause unusual lesions in the skin .One of the most striking is the appearance of clean linear erosions in skin creases, with appearance of a knife cut[49].Herpetic sycosis is a recurrent or initial herpes simplex infection affecting primarily the hair follicles[50].Eczema herpeticum is an infection with herpes virus with chronic atopic dermatitis may result in spread of herpes simplex throughout the eczematous areas[50].The exact cause of Bell's palsy, a type of facial paralysis, is unknown, it may be related to reactivation of HSV-1[51].The theory has been contested, however, since HSV is detected in large numbers of individuals having never experienced facial paralysis, and higher levels of antibodies for HSV are not found in HSV-infected individuals with Bell's palsy compared to those without[52].

\section{Diagnosis}

Primary orofacial herpes is readily identified by clinical examination of persons with no previous history of lesions and contact with an individual with known HSV-1 infection. The appearance and distribution of sores in these individuals typically presents as multiple, round, superficial oral ulcers accompanied by acute gingivitis[53].Genital herpes can be more difficult to diagnose than oral herpes, since HSV-2 infected persons have no classical symptoms[53].Laboratory tests include culture of the virus, direct fluorescent anti body(DFA) studies to direct virus, skin biopsy, and polymerase chain reaction test for presence of viral DNA[53].Laboratory confirmation permits subtyping of the virus, which may help predict frequency of reactivation after first-episode oral or genital HSV infection, site of CNS infection, and likelihood of drug resistance[54].Until recently, serological tests for antibodies to HSV were rarely useful to diagnosis and not routinely used in clinical 
practice[53].The older IgM serologic assay could not differentiate between antibodies generated in response to HSV-1 or HSV-2 infection. However, the new immunodot glycoprotein G-specific(IgG) HSV test is more than $98 \%$ specific at discriminating HSV-1 from HSV-2[9].Some modern medical professionals believe the new IgG test should always be clinically preferred to the old IgM test, but not all doctors appeared to be informed of the availability the newer, reliable IgG test[55].

\section{Management and therapy}

No method eradicates herpes virus from the body, but antiviral medications can reduce the frequency, duration and severity of outbreaks. Analgesics such as ibuprofen and paracetamol (acetaminophen) can reduce pain and fever. Topical anesthetic treatment such as prilocaine, lidocaine, benzocaine, or tetracaine can also relieve itching and pain [10].

Several antiviral drugs are effective for treating herpes, including acyclovir, valaciclovir (valacyclovir),famiciclovir,andpenciclovir.Acyclovir was the first discovered and is now available in generic[56].Evidence supports the use of acyclovir and valacyclovir in the treatment of herpes labialis as well as herpes infections in people with cancer [57, 58].The evidence to support the use of acyclovir in primary herpetic gingivostomatitis is weaker[59].Numerous trials of acyclovir in mucocutaneous HSV infections of the immunocompetent and immunosuppressed hosts have been conducted [60]. Famciclovir, the oral formulation penciclovir,is also clinically effective in the treatment of a variety of HSV-1 and HSV-2 infections [61].Valacylovir is a valyl ester of acyclovir that has greater bioavailability[62]. A number of topical antiviral are effective for herpes labialis,includingacyclovir,penciclovir and docosano[57].

Certain alternative(complimentary) medicines are claimed to be beneficial in the treatment of herpes that include echinacea,eleuthro,L-lysine,zinc,monolaurin bee products,andallovera,evidence is insufficient though to support use of many these compounds[63].

\section{Prevention and Prognosis}

As with almost all sexually transmitted infections, women are more susceptible to acquiring genital HSV-2 than men [64].On an annual basis, without the use of antiviral or condoms, the transmission risk of HSV-2 from infected male to female is about 8-11\%[65]..This believed to be due to the increased exposure of mucosal tissue to potential infection sites. The transmission risk from infected female to male around 4-5\% annually[65].Suppressive antiviral therapy reduces these risks by 50\%[66]. Antiviral also help prevent the development of symptomatic HSV in infection scenarios, meaning the infected partner will be seropositive but symptom-free by around 50\%.Condome use also reduces the transmission risk significantly[67].Condom use is much more effective at preventing male-to-female transmission than vice versa[67].The effects of combining antiviral and condom use is roughly additive, thus resulting in a $75 \%$ combined reduction in annual transmission risk. Previous HSV-1 infection appears to reduce the risk for acquisition of HSV-2 infection among women by factor of three [68].However, asymptomatic carriers of the HSV-2 are still contagious. Some asymptomatic individuals are unaware of their infection, they are considered at high risk for spreading HSV[69]. When one partner has herpes simplex infection and other does not, the use of antiviral medication, such as valaciclovir,in conjunction with a condom, further decreases the chances of transmission to uninfected partner[27].In 0ctober 2011, the anti-HIV drug tenofovir, when used topically in a microbial vaginal gel was reported to reduce herpes virus sexual transmission by $51 \%[70]$.

The risk of transmission from mother to baby is highest if mother becomes infected around the time of delivery $(30 \%$ to $60 \%$ ) since insufficient time will have occurred for the generation and transfer of protective maternal antibodies before the both of child. In contrast, the risk falls to $3 \%$ if the infection is recurrent [71,72].Acyclovir is the recommended antiviral for herpes suppressive therapy during the last months of pregnancy. The use of valaciclovir and famciclovir,while potentially improving compliance, have less-welldetermined safety in pregnancy[27].

\section{Prognosis}

Following active infection, herpes viruses establish a latent infection in sensory and autonomic ganglia of the nervous system. The double -stranded DNA of the virus is incorporated into the cell physiology by infection of the nucleus of nerve's cell body .HSV latency is static; no virus is produced, and is controlled by a number of viral genes, including latency-associated transcript [73].Many HSV-infected people experience recurrence within the first year of infection[27].Prodromeprocedes development of lesions.Prodromal symptoms include tingling (paresthesia),itching and pain where lumbosacral nerves innervate the skin.Prodrome may occur as long as several days or as short as a few hours before lesion develop[27]. The causes of reactivation are uncertain, but several potential triggers have been documented. A 2009 study showed the protein VP1esthesia),itiching and pain where lumbosacral nerves innervate the skin .Prodrome may occur as long as several days or as short as a few hours before lesion develop[27].The causes of reactivation are uncertain, but 
several potential triggers have been documented. A 2009 study showed the protein VP16 plays role in reactivation of the dormant virus[74].The changes in immune system during menstruation may play a role in HSV-1 infection[75].Other triggers include local injury to the face, lips, or mouth, and exposure to ultraviolet light[76,77].

The frequency and severity of recurrent outbreaks vary greatly between people. Some individuals' outbreaks can be quite debilitating, with large, painful lesions persisting for several weeks, while others experience only minor itching or burning for a few days. Some evidence indicates genetics play a role in the frequency of cold sore outbreaks .Antiviral medication has been proven to shorten the frequency and duration of outbreaks[78].In the case of a genital infection, sores can appear at the original site of infection or near the base of the spine, the buttocks, or the back of the thighs.HSV-2 infected individuals are at high risk for acquiring HIV when practicing unprotected sex with HIV-positive persons, in particular during an outbreak with active lesions[79].

\section{Future directions}

Extensive research has gone into vaccines for both prevention and treatment of herpes infections. Unsuccessful clinical trials have been conducted for glycoprotein subunit vaccines. For therapy, only a single replication-competent HSV vaccine has undergone testing, a competent vaccine proposals require further animal testing [80].Researchers at the University of Florida have made a hammerhead ribozyme that targets and cleaves the mRNA of essential genes in HSV-1.The hammerhead, which targets the mRNA of the UL20 gene, greatly reduced the level of HSV-1 ocular infection in rabbits, and reduced the viral yield in vivo,but further research is required before it can be attempted in people infected with herpes. [81,82].Another group of researchers at Duke University pursued another possibility to eradicate the HSV-1 variant, by figuring out how to switch all copies of the virus in the from latency to their active stage at the same time, rather than the way the virus copies normally stagger their activity stage, leaving some dormant somewhere at allimes, congenital antiviral drugs might kill the entire virus population, since they can no longer hide in the nerve cells. One class of drug called antagomir could serve this purpose[83]. Other researcher believe a drug could be developed to block the microRNA whose job is it to suppress HSV-1 into latency[84].A genomic study of the herpes simplex type-1 virus confirmed the human migration pattern theory called 'out-of-Africa' hypothesis[85].

\section{Conclusion}

Herpes simplex virus(HSV) infections are prevalent worldwide.HSV-1 causes mainly mouth, throat, face, eye, and central nervous system infections,HSV-2 causes primarily genital infections. Recurrent outbreaks are common especially among immunosuppressed persons.Prevention by use of condomand antiviral drugs.

\section{References}

[1]. Mertz KJ,TreesD,LevineWC,etal.Etiology of gentital ulcers and prevalence of human immunodeficiency virus coinfection in 10 US cities.TheGenital Ulcer Disease Surveillance Group.J Infect Dis.1998;178:1795-98.

[2]. ChayavichitsilpP,BuckwalterJV,KrakowskiAC,etal.Herpessimplex.PediatrRev.2009;30(4):119-29.

[3]. Looker KJ,GarnettGP,SchmidGP.An estimate of the global prevalence and incidence of herpes simplex virus type 2 infection \{http:// ncbi. nlm. nih.gov/ pmc/articles/PMC2649511).Bulletin of the World Health Organization. 2008; 86(10):805-12,A.

[4]. Looker KJ,GarnettGP,SchmidGP.An estimate of the global prevalence and incidence of herpes simplex virus type 2 infection http:// www. int/bulletin/ volumes/86/10/07-046128/en).Bull.World Health Organ.2008;86(10):805-12,A.

[5]. Xu Fujie,FujieXu.MayaR,etal.Trends in Herpes simplex Virus Type 1 and Type 2 Seroprevalence in the United States.JAMA.2006;296(8):964-73.

[6]. CDC Study Finds U.S. Herpes Rates Remain High (Http:// www.cdc .gov/nchhstp/newsroom/hsv2pressrelease.html).Center for Disease Control and Prevention.2010-03-09.Retrieved 2012-02-19.

[7]. AHMF:Prevention $\quad$ Sexual $\quad$ Transmission $\quad$ of Genital web.archive.org/web/20080121164311http://www.ahmf.au/health_professionals/guidelines/preventing_gh_transmission.htm).2008. Retrieved 2008-02-24.

[8]. Dickerson FB,BorrowJJ,StallingC,etal.Infection with herpes simplex virus type 1 is associated with cognitive deficit in bipolar disorder.Biol Psychiatry.2004;55(6):588-93.

[9]. Ashley RL,etal.Comparison of Western blot(immunodot) and glycoprotein G-specific immunodot enzyme assay for the detection antibodies to herpes simplex virus type 1 and type 2 in human sera.JClin Microbiol.1988;26(4):662-7.

[10]. 0’Mahony C,TimmsMS,RamsdenST.Local anesthetic creams. BMJ.1988; 297 (6661):1468.

[11]. WidyP.Herpes history and classification. In Kaplan A,ed.The Herpes viruses.NewYork:Academic Press;1973;1-25.

[12]. John Leo(1982-08-02)The New Scarlet Letter (http:www. time.com/ time/ magazine/ article/0,9171,1715020,00.htlm). Time.

[13]. AstrucJ.DeMorbisVenereisLibri Sex. Paris.1736.

[14]. Parker F,NyeR.Studies on filterable viruses 11.Cultivation of herpes virus.Am JPathol.1925;1:37.

[15]. NahmiasAJ,DowdleWR.Antigenic and biologic differences in herpesvirus hominis.Prog Med Virol.1968;10:110-159.

[16]. Chow AW,RolandA,FialaM,etal.CytosineArabinoside Therapy for Herpes Simplex Encephalitis-Clinical Experience with Six Patients.AntimicrobAgentsChemother. 1973;3(3):412-7.

[17]. Kaufman HE,HowardGM.Therapy of experimental herpes simplex keratitis.Invest 0 phthamol.1962;1:561-4.

[18]. Ch'ienLt,WhitleyRJ.AlfordCA,etal.Adeninearabinoside for therapy of herpes zoster in immunosuppressed patients:preliminary results of a collaborative study.J InfectDis.1976;133(Suppl):A184-91. 
[19]. McKelveyEM,KwaanHC.Cytosinearabinoside therapy for disseminated herpes zoster in a patient with IgG pyroglobulinemia.Blood.1969;34(5):706-11.

[20]. NahmiasAJ,KibrickS.Inhibitory Effect of Heparin in Herpes Simplex virus.JBacteriol.1964;87(5):1060-6.

[21]. 0'Meara A,DeasyPF,HillaryIB,etal.Acyclovir for treatment of mucocutaneous herpes infection in a child with leukaemia.Lancet.1979;2(8153):1196.

[22]. Whitley R,ArvinA,ProberC,etal.A controlled trial comparing vidarabine with acyclovir in neonatal herpes simplex virus infection.Infectious Diseases Collaborative Antiviral Study Group.N.Engl J Med.1991;324(7):444-9.

[23]. KimberlinDW,LinCY,JacobsRF,etal.Safety and efficacy of high- dose intravenous acyclovir in the management of neonatal herpes simplex virus infections. Pediatrics. 2001;108(2):230-8.

[24]. MayouR.Psychological morbidity in a clinic for sexually transmitted disease.Br JVenereal Dis.1975;51(1):57-60.

[25]. VeizinaC,StebenM.GenitalHerpes:Psychosexual Impacts and Councelling.Canad JCME(June).2001;125-34.

[26]. DecmanV,KinchingtonPR,HarveySA,etal.Gamma interferon can block herpes simplex virus type 1 reactivation from latency even the presence of late gene expression.J Virol.2005;79:10339-47.

[27]. LipschutzB.Untersuchungenuber die Aetologie der Krankheiten der Herpes Gruppe(herpes zoster genitalis. Herpes fibrillis)ArchDermatolSymp(Berl).1921;136:428.

[28]. WalidA,ZehJ,SelkeS, et al. Reactivation of genital herpes simplex virus type 2 infection in asymptomatic seropositive persons. N.Engl J Med.2000;342:844-850.

[29]. Perl TM,HaugenTH,PfallerMA,etal.Transmission of herpes simplex virus type 1 infection in an intensive care unit.Am Intern Med.1992;117:584-6.

[30]. CherpesTL,MeynLA.HillerSL.Cunnilingus and vaginal intercourse are risk factors for herpes simples type 1 acquisition in women. Sex Transm Dis.2005;32:84-89.

[31]. BelongiaEA,GoodmanJL,HollandEJ,et al. An outbreak of herpes gladiatorum at a high school wrestling camp.NEngl $J$ Med.1991;325:906-10.

[32]. Brown ZA,SelleS,ZehJ,etal.The acquisition of herpes simplex virus during pregnancy.NEngl J Med.1997;337:509-13.

[33]. QuninTC,CoreyL,ChatfeeRG,etal.The etiology of anorectal infection in homosexual men.Am J Med.1981;71:395-406.

[34]. Gray R,WawerM,BrookmyerR,etal.Probability of HIV-1 transmission per coital act in monogamous heterosexual HIV-1 discordant couple in Rakai Uganda. Lancet. .2001;357:1149-53.

[35]. Sacks Sl,GriffithsPD,CoreyJ,etal.Introduction: is viral shedding a surrogate marker for transmission of genital herpes?.Antiviral Res.2004;63(Suppl 1):S3-S9.

[36]. Mark KE,WaldA,MargaretAS, et al. Rapidly cleared episode of herpes simplex virus reactivation in immunocompetent adults.J Infect Dis.2008;198:1141-49.

[37]. Blower S,Wald,A,GershengornH,etal.Targetingvirological core groups: a new paradigm for comtrolling herpes simples virus type 2 epidemic.J InfectDis.2004;190:1610-17.

[38]. Stanberry LR,KernER,RichardsJT, etal.Genital herpes in guinea pigs:pathogenesis of primary infection and description of recurrent disease .J Infect Dis.1982;146:397-404.

[39]. Rock DL,FraserNW.Detection ofHSV-1 genome in central nervous system of latently infected mice.Nature.1983;302:523-15.

[40]. Cushing H.surgical aspects of major neuralgia of trigeminal nerve: report of 20 operation upon the gasserian ganglion with autonomic and pathologic notes on the consequences of its removal .JAMA.1905;44:773-79.

[41]. Baringer JR, Recovery of herpes simplex virus from human sacral ganglion.NEngl JMed.1974;291:828-30.

[42]. Corey J, Spear PG.Infection of herpes simplex virues(1).N Engl J Med.1986;314:686-91.

[43]. Johnson C,MargaretA,SelleS, etal.Herpes simplex virus viremia during primary genital infection.J Infect Dis.2008;198:31-34.

[44]. CrespiC,CumberlandW,WaldA,etal.Longitudnal study of herpes simplex virus type 2 infection using viral dynamic modelelin.SexTransm Infect.2007;83:359-64.

[45]. KusneS,SchwartzM,BreinigMK, et al. Herpes simplex virus hepatitis after solid organ transplantation in adults.J Infect Dis.1991;163:1001-1007.

[46]. Mertz GJ.Epidemiology of genital herpes infections.Infect Dis Clin NorthAm.1993;7(4):825-39.

[47]. http://www.ncbi.nlm.gov/pmc/articles/PMC164775/

[48]. HandsfieldHH.Public Health Strategies to Prevent Genital Herpes:Where Do We Stand?.Curr Infect Dis Rep.2000;2(1):25-30.

[49]. OcelynA,LiebSB,BrismanSH,etal.Linear erosive Herpes Simplex Virus infections in immunocompromised patients: the knife cut sign. Clin Infect Dis.2008.47(11):1440-1.

[50]. James WD,BergerTG.Andrews' Diseases of the Skin clinical Dermatology. SaundersElsevier.2006.ISBN 0-7216-2921-0.

[51]. TankereF,Bernat I. Bell's palsy:fromaetiology to diagnostic reality. Rev.MedInterne(in French).2009;30(9):769-75.

[52]. Linder T,BossartW,BodmerD.Bell's palsy and Herpes simplex virus: fact or mystery ?.Otol Neurotol.2005;26(1):109-13.

[53]. FatazadehM,SchwartzRA.Herpes simplex virus infections: epidemiology, pathogenesis symptomatology, diagnosis, and management.J.AmAcadDermatol. 2007; 75 (5):737-63;quiz764-6.

[54]. Corey L,Huang ML, SElleS,etal.Diferentiation of herpes simplex virus type 1 and 2 in clinical samples by a real time taqman PCR assay.J Med Virol.2005;76:350-55.

[55]. Warren T. The Good News About The Bad News(Http://books.com.Harbinger Publications.p.209.ISBN 1-557224-618-9.

[56]. LaFemina,edited by RoberL.Antiviralresearch:strategies in antiviral drugdiscovery.Washington,DC:ASM Press.2009;p.1.ISBN 978-1-55581-439-7.

[57]. Chon T,NguyenL,ElliottTC.Clinicalinquiries. What are the best treatments for herpes labialis ?.J Fam Pract.2007;56(7):576-8.

[58]. Glenny AM,FernandezMauleffinchIM,PavinS,etal.ed.Interventions for the prevention and treatment of herpes simplex virus in patients being treated for cancer.Cochrane Database Sys Rev.2009;(1):CD006706.

[59]. Nasser M,Fedorowicz Z, KoshnevisanMH,etal.Monaed.Acyclovir for treating primary gingivostomatitis.Cochrane Database Syst Rev.2008;(4):CD006700.

[60]. Wald A,CarrelD,RemingtonM,etal.Two day regimen of acyclovir for treatment of recurrent genital herpes simplex virus type 2 infection.Clin Infect Dis.2002;34:944-48.

[61]. Bartlett BL,TyringSK,FifeK,etal.Famciclovir treatment options for patients with frequent outbreaks of recurrent genital herpes the RELIEF trial.JClinVirol.2008;43:190-95.

[62]. LaiskonisA,ThuneT,NeldamS,etal.Valacyclovir in the treatment of facial herpes simplex virus infection.J Infect Dis.2002;186(Supp11)S66-S70.

[63]. Reviewed by EBSCO CAM Reviewed Board in July 2012.(http://healthlibraryepnet.com/print.aspx?token=deh453e6-8aa2-4e28b56c-5e30699d7b3c\&ChunkIID=21570).HERPES:Principal Proposed Natural Treatment I Other Proposed Natural Treatments.EBSCO.Retrieved 11 May 2013. 
[64]. Carla K J.Percentage of people with herpes drops org/web/20120318125331/http:www.aegis.com/news/ap/2006ap060840.html).

[65]. KulhanjianJA,SoroushV,AuDS,etal.Identification of women at unsuspected risk of primary infection with herpes virus type 2 during pregnancy.NEnglJMed. 1992; 326(14):916-20.

[66]. Corey L,WaldA,PatelR,etal.Once daily valacyclovir to reduce risk of transmission of genital herpes.NEng J Med.2004;350(1):1120.

[67]. Wald A,LagenbergAG,KrantzE,etal.The relationship between condom use and herpes simplex virus acquisition. Annals of Internal Med.2005;143(1):707-13.

[68]. Mertz GJ,BenedettiJ,AshleyR,etal.Risk factors for the sexual transmission of genital herpes.Annals of Internal Med.1992;116(3):197-202.

[69]. Genital Herpes-CDC Fact Sheet(http://www.cdc.gov/std/herpes/STDFact-herpews details.htm\#ref21).Center for Disease Control and Prevention.Retrieved 2014-01-30.

[70]. McNeilDJ.http://www.nytimes.com/2011/10/21/health/research/21herpes.htmlTopicalTenofovir,Microbicide Effective against HIV,inhibits Herpes Simplex Virus-2-Replication.NY Times Research Article.

[71]. Brown ZA,SelkeS,ZehJ,etal.The acquisition of herpes simplex virus during pregnancy.NEngl J Med.1997;337(8):509-15.

[72]. Brown ZA,BenedettiJ,AshleyR,etal.Neonatal herpes simplex virus infection in relation to asymptomatic maternal infection at of labor.NEnglJMed. 1991; 324(18):1247-52.

[73]. StumpfMP,LaidlawZ,JansenVA.Herpes viruses hedge their bets.Proc Natl AcadSci.U.S.A.2002;99(23):15234-7.

[74]. How Herpes Re-rears Its Ugly Head. (Http:www. Sciencenews .org/ view. org.view.generic/id/42223/title How_herpes_rerears_ugly_head)Science News.

[75]. MysliwskaJ,TrzonkowskiP,BrylE,etal.Lower interleukin12 and higher serum tumor necrosis factor-a levels are associated with premenstrual ,recurrent facial herpes simplex infection in young women.Eur Cytokine News. 2000;11(3):397-406.

[76]. Chambers A,Perry M. Salivary mediated autoinoculation of herpes simplex virus on the face in the absence of "cold sores" after trauma. J Oral MaxillofacSurg.2008; 66(1):136-8.

[77]. Perna JJ, MannixML,RooneyJF, etal.Reactivation of latent herpes simplex virus infection by ultraviolet light: a human model.J Am Acad Dermatol.1987;17(3):473-8.

[78]. Martinez V,CaumesE,Chosidow 0.Treatment to prevent recurrent genital herpes.Current Opin Infect Dis.2008;21(1)42-48.

[79]. KoellDM,CoreyL.HerpesSimples:Insights on Pathogenesis and Possible Vaccines. Annu Rev Med.2008;59:381-95.

[80]. Johnston C,KoelleDM,Wald A.HSV-2:in pursuit of a vaccine.JClinInvest. 2011; 121(12):4600-9.

[81]. Liu J,TuliSS,BloomDC,etal.Gene Therapy Targeting Herpes Simplex Virus. Molecular Therapy.2006;13:S310

[82]. Potential new herpes therapy studied.(Http://news.ufl.edu/2009/02/03/herpes-2/).University Florida News.2009-02-03.Retrieved 2011-04-12.

[83]. Fox Maggie.New approach chance to finally herpes.(http//www.reuters.com/article/idUSN0229815620080702?pageNumber=2\&virtualBrandChannel=0).Reuters.Retrieved 2011-04-12.

[84]. Kingsbury K.A Cure for Cold Sores? .(http://www .time. com/time /health /article/ 0, 8599.1819739,00.htlm).Time.Retrieved 201005-04.

[85]. Foley JA.Hitchhiking Herpes Virus Aligns with spread of Human Civilization. NatureWorldNews.com.Retrieved 22 0ctober 2013. 